\title{
"I had Made the Decision, and No One was Going to Stop Me" -Facilitators of PrEP Adherence During Pregnancy and Postpartum in Cape Town, South Africa
}

\author{
Dvora L. Joseph Davey ${ }^{1,2}$ - Lucia Knightt ${ }^{3,4}$. Jackie Markt-Maloney' ${ }^{1}$. Nokwazi Tsawe ${ }^{2}$. Yolanda Gomba ${ }^{2}$. \\ Nyiko Mashele ${ }^{2} \cdot$ Kathryn Dovel $^{6} \cdot$ Pamina Gorbach $^{1} \cdot$ Linda-Gail Bekker $^{5} \cdot$ Thomas J. Coates $^{6} \cdot$ Landon Myer $^{2}$
}

Accepted: 22 May 2021 / Published online: 3 June 2021

(c) The Author(s), under exclusive licence to Springer Science+Business Media, LLC, part of Springer Nature 2021

\begin{abstract}
PrEP is safe and effective but requires adherence during potential HIV exposure, yet the facilitators of long-term maternal adherence are not well understood. We conducted semi-structured interviews with 25 postpartum women who reported high adherence (PrEP use $\geq 25$ days in last 30-days and never missed a PrEP prescription in pregnancy/postpartum period) within a PrEP service for pregnant and postpartum women. A thematic approach guided an iterative process of coding and analysis. Themes identified as drivers of optimal PrEP use were HIV risk perception, mainly because of partner's behaviors and unknown serostatus, and a strong desire to have a baby free of HIV. Reported disclosure of PrEP use facilitated PrEP adherence. Women discussed having partner and family support, which included reminders to take PrEP daily. Primary barriers were anticipated or experienced stigma, overcome through education of partners and family about PrEP. Pregnant women experienced transient side-effects, but found ways to continue, including taking PrEP at night. PrEP programs for pregnant and postpartum women should integrate strategies to assist women with realistic appraisals of risk and teach skills for disclosure and securing support from significant others.
\end{abstract}

Keywords HIV prevention $\cdot$ Pregnancy $\cdot$ Pre-exposure prophylaxis $\cdot$ PrEP $\cdot$ Postpartum $\cdot$ South Africa

\section{Resumen}

La profilaxis Pre-exposición (PrEP, siglas en inglés) es segura y eficaz, pero requiere adherencia durante una posible exposición al VIH; sin embargo, no se conocen bien los factores que facilitan la adherencia materna a largo plazo. Realizamos entrevistas semiestructuradas con 25 mujeres en posparto que informaron un alto cumplimiento (uso de PrEP $>25$ días en los últimos 30 días y nunca omitieron una receta de PrEP en el embarazo y período posparto) dentro de un servicio de PrEP para mujeres embarazadas y posparto. Un enfoque temático guio un proceso iterativo de codificación y análisis. Los temas identificados como impulsores del uso óptimo de la PrEP fueron la percepción del riesgo de VIH, principalmente debido a los comportamientos de la pareja y el estado serológico desconocido, y un fuerte deseo de tener un bebé libre de VIH. La divulgación informada del uso de PrEP facilitó la adherencia a la PrEP. Las mujeres hablaron sobre el apoyo de su pareja y

Dvora L. Joseph Davey

dvoradavey@ucla.edu

1 Department of Epidemiology, Fielding School of Public Health, University of California Los Angeles, 615 E Charles Young Drive S, Los Angeles, CA 90095, USA

2 Division of Epidemiology and Biostatistics, School of Public Health and Family Medicine, University of Cape Town, Cape Town, South Africa

3 Division of Social and Behavioural Sciences, School of Public Health and Family Medicine, University of Cape Town, Cape Town, South Africa
4 School of Public Health, University of the Western Cape, Bellville, South Africa

5 The Desmond Tutu Health Foundation, University of Cape Town, Cape Town, South Africa

6 Division of Infectious Diseases, David Geffen School of Medicine, University of California Los Angeles, Los Angeles, CA, USA 
familia, incluidos recordatorios para tomar la PrEP cada dia. Las barreras primarias fueron el estigma anticipado o experimentado, superado a través de la educación de los socios y familiares sobre la PrEP. Las mujeres embarazadas experimentaron efectos secundarios transitorios, pero encontraron formas de continuar, incluida la toma de PrEP por la noche. Los programas de PrEP para mujeres embarazadas y posparto deben integrar estrategias para ayudar a las mujeres con evaluaciones realistas del riesgo y enseñar habilidades para la divulgación y obtener el apoyo de otras personas importantes.

\section{Introduction}

Women in sub-Saharan Africa face a high risk of HIV acquisition during pregnancy and breastfeeding [1]. While services to eliminate mother-to-child HIV transmission (EMTCT) have expanded rapidly in the region, few primary prevention interventions exist for pregnant women who initially test HIV-negative. This is a missed opportunity with implications for the woman, but also her partner and infant $[2,3]$. Acute HIV infection in pregnancy and postpartum increases the risk of vertical transmission, accounting for an estimated third of vertical HIV transmissions $[4,5]$. To protect women and contribute to EMTCT, the World Health Organization (WHO) recommends offering pre-exposure prophylaxis (PrEP) to pregnant and postpartum women at risk of HIV acquisition [6-8]. In South Africa, an estimated 76,000 infant HIV cases are expected between 2020 and 2030 , though this could reduce significantly if pregnant women initiate and persist on PrEP [9].

A recent systematic review provides no safety-related rationale for prohibiting PrEP during pregnancy and/or breastfeeding [10]. While safety data are reassuring, recent pharmacokinetic studies reveal that tenofovir diphosphate concentrations were one-third lower in pregnancy compared to postpartum women, highlighting how essential daily PrEP use is in pregnant women [11, 12]. There are numerous barriers to optimal daily, long-term PrEP use in pregnant and postpartum women across health facility-, interpersonal and intrapersonal-levels [10, 13, 14] [15].

Though longer acting PrEP agents like rings or injectables will change the adherence issues associated with daily oral PrEP, these longer acting PrEP agents may be many years away from regulatory approval for use in pregnant and lactating women $[16,17]$. Therefore, daily oral PrEP may be the only PrEP option available for pregnant and postpartum women for quite some time. Further, similar to other populations, pregnant and postpartum women will need a choice between longer acting and daily PrEP, highlighting the need to identify characteristics associated with longer-term daily oral use in this population.

Within a larger study of PrEP in pregnancy, we conducted in-depth interviews with postpartum women who started PrEP in pregnancy and reported high levels of adherence though the transition to the postpartum period. The aim of this study is to understand the positive drivers of PrEP initiation and adherence in pregnant and postpartum women to inform future interventions to optimize PrEP use in this vulnerable population.

\section{Methods}

The PrEP in pregnancy and postpartum (PrEP-PP) study is a prospective cohort enrolling adolescent girls and women at one primary care, public health clinic in Cape Town, South Africa (NCT03902418) [18]. Participants are enrolled at their first antenatal care (ANC) visit and followed participants through 12-months postpartum. Inclusion criteria include being: $(1)=>16$ years old, (2) confirmed HIVnegative (3) intending to stay in Cape Town, (4) confirmed pregnancy status, and (5) absence of medical contraindications to PrEP. Following the baseline survey, women are counselled about the risk of HIV in pregnancy and postpartum, including the benefits and risks of taking PrEP. If women wish to start PrEP, nurses prescribe a one-month supply of Truvada ${ }^{\circledR}$ (tenofovir disoproxil fumarate/emtricitabine). Participants on PrEP receive an invitation card to return for HIV testing and counselling, refill prescriptions and adherence counselling.

For this qualitative sub-study, we purposively recruited for homogeneity twenty-five postpartum women between July and September, 2020. Enrolment was from the subset of women in the PrEP-PP cohort who were: (1) in study for $3+$ months, (2) delivered a live infant, (3) received regular PrEP prescriptions in pregnancy and postpartum period, and (4) reported high PrEP adherence in pregnancy and postpartum (defined as $=>25$ of last 30 days on PrEP). Consenting participants received 120 Rand (approximately \$8 USD) in grocery vouchers for their time and transport costs at each study visit.

One female, isiXhosa speaking Interviewer (NT, MPH) conducted one semi-structured interview with eligible participants in the local language (isiXhosa). The Interviewer had received training in qualitative interviews prior to the study and had two years' experience in conducting qualitative research. The Interviewer was not part of the main PrEP-PP study and did not have any relationships with the participants prior to the interview. No characteristics were reported about the interviewer to the participants. 
Participants were interviewed telephonically due to the COVID-19 lockdown and associated risk $(n=19$ interviews), or in a private room within the health facility ( $\mathrm{n}=6$ interviews; both participant and interviewer wore masks and sat $>1 \mathrm{~m}$ apart). Interviews lasted approximately $30-40 \mathrm{~min}$. Interviews were audio-recorded along with field notes after the interview. Interviews were translated into English, and transcribed by one trained research staff (YM, MPH). The Interviewer (NT) reviewed all transcriptions and translations for accuracy and made necessary corrections.

A thematic approach guided an iterative process of coding and data analysis in NVivo 12 (QSR International, Victoria, Australia) [19]. The analysis integrated memo writing and the concept mapping to refine understandings of PrEP adherence and develop themes that facilitated adherence. Three trained research assistants (YG, JMM, NT) conducted the analysis led by the study PI (DJD). The research assistants are females who are from the local community (2 MPH educated) and one from UCLA (MPH student). All of the Research Assistants had received training on qualitative coding and co-developed the codebook from the study's theoretical framework. The PI of the study, DJD, reviewed the results. The Consolidated Criteria for Reporting Qualitative Research (COREQ) checklist guided the analysis and reporting [20].

\section{Conceptual Framework}

We adapted Ickovics' and Meisler's conceptual framework on factors affecting HIV treatment adherence [21] that was used $[22,23]$ to situate and organise the findings into the broader context of the intersection between individual women and biomedical interventions ahead of the interviews
(Fig. 1). The conceptual framework considered: (1) individual, (2) HIV factors, (3) pill safety/side-effects, and (4) facility-level factors explored from the participants' perspective. The interview guide was developed, and pre-tested prior to the study, to explore these factors in more depth.

\section{Ethics}

The study was approved by the University of Cape Town (\#297/2018) and the University of California Los Angeles Institutional Review Board (IRB\#18-001622). All participants provided written informed consent during study enrolment.

\section{Results}

\section{Participant Characteristics}

Participants were 16 to 43 years old and had been on PrEP for a median of 9 months. Almost all participants reported vaginal sex during pregnancy, fewer than one-third were married or cohabiting with a partner, and several reported multiple sex partners in the past year (Table 1).

\section{Individual Factors}

Women reported various reasons for why they initiated PrEP in pregnancy, as well as why they persisted on PrEP during pregnancy and the postpartum period. Most women decided to start PrEP to protect their baby from HIV. After taking PrEP for months, women reported not wanting to "go

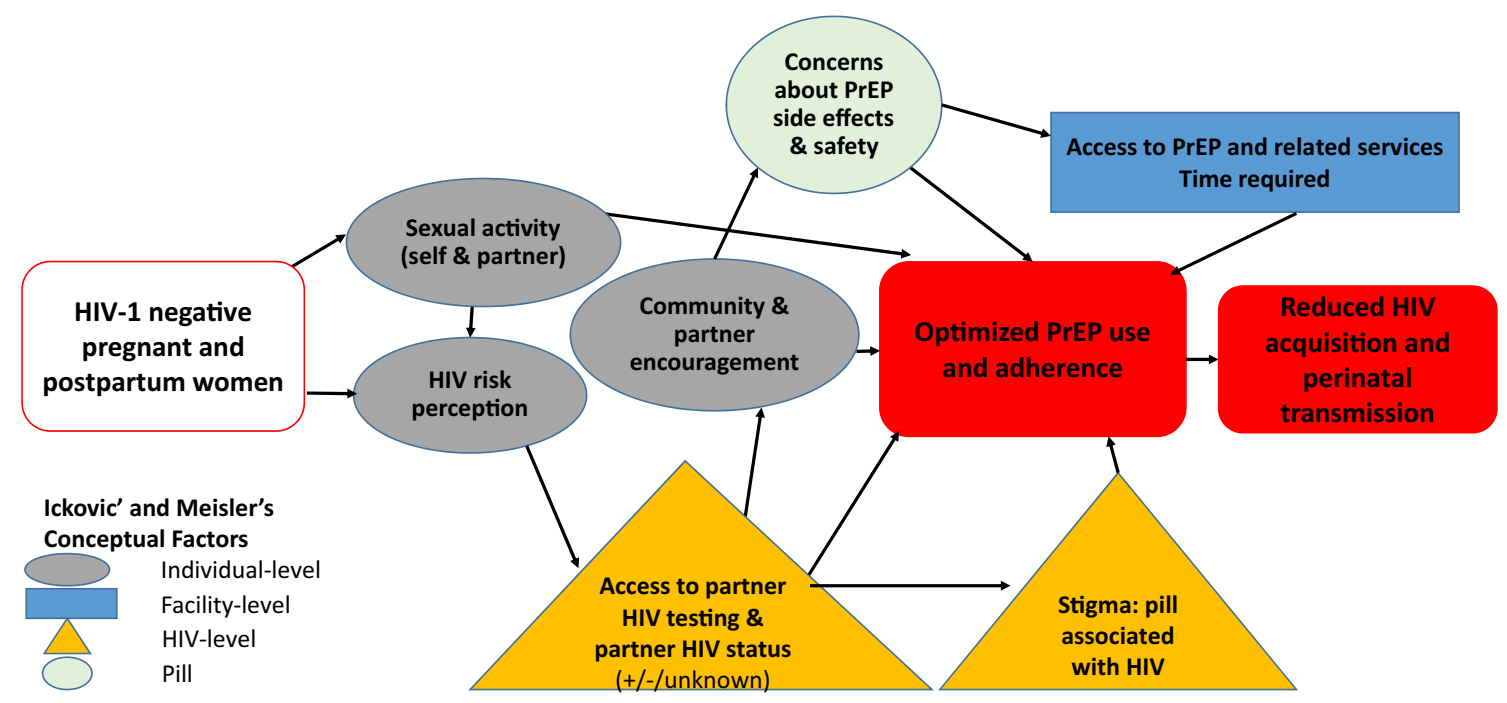

Fig. 1 PrEP-PP conceptual model of individual, disease, facility level and pill factors associated with PrEP adherence adapted from Ickovics \& Meisler [21] 
backwards" and being at risk of acquiring HIV again. One woman said, "I had made the decision, and no one was going to stop me. I was not going to go backwards" (participant identifier [PID] PID005, 24 years). Others reported a feeling of relief or happiness while taking PrEP because they felt protected against HIV and "safe" during pregnancy. Below we present specific factors that encouraged women to start and stay on PrEP.

Women reported a period of postpartum abstinence ranging from weeks to months after giving birth. Despite this, the enrolled women who reported high adherence did not report stopping PrEP in this time. As one woman noted: "It [taking PrEP] was routine. I was not forgetting to take it anymore. Stopping or taking a break never came to mind." (PID005, 24 years) Several women mentioned having more than one sex partner during pregnancy and postpartum and that PrEP helped them feel safer, especially with condomless sex.
Motivation of HIV-Free Infant A desire to remain HIV uninfected and have an HIV-free infant were strong motivators to continue PrEP.

It [PrEP] is protecting the unborn baby. It was very important that I protect the baby. I knew I was (HIV) negative, so I didn't know what the situation would be when the baby came. So, I was interested [in PrEP] because I wanted nothing [to happen] as I can see the conditions of people who are infected. What was important was protection, then I made the decision. (PID067, 41 years)

This focus on protecting the baby was clearly presented and reinforced by the project staff during enrolment and influenced women's thinking, for example: "They [project staff] explained that when you are breastfeeding, it is important to take these pills because they protect the baby. I plan on continuing because I am still breastfeeding." (PID152, 34 years).

Table 1 Sociodemographic characteristics of interview participants with high self-reported PrEP adherence in the PrEP-PP study ( $\mathrm{n}=25$ ), JulySeptember, 2020

\begin{tabular}{|c|c|c|}
\hline & Median & IQR \\
\hline Maternal age (in years) & 26 & 24,34 \\
\hline Gestational age in weeks (at baseline PrEP-PP study enrolment) & 24 & 19,31 \\
\hline Weeks postpartum (at interview) & 18 & 7,24 \\
\hline Time in PrEP-PP study (at interview, in weeks) & 36 & 33,36 \\
\hline \multirow[t]{2}{*}{ Number of previous pregnancies } & 2 & 1,3 \\
\hline & $\mathrm{N}$ & $\%$ \\
\hline \multicolumn{3}{|l|}{ Educational level } \\
\hline Below secondary school & 14 & 56 \\
\hline Completed secondary school & 9 & 36 \\
\hline Some tertiary education & 2 & 8 \\
\hline \multicolumn{3}{|l|}{ Relationship status with father of child } \\
\hline No relationship & 3 & 12 \\
\hline Married & 2 & 8 \\
\hline Steady partner living with me & 5 & 20 \\
\hline Steady partner not living with me & 15 & 60 \\
\hline \multicolumn{3}{|l|}{ Employment status } \\
\hline Employed full or part-time & 7 & 28 \\
\hline Student & 2 & 8 \\
\hline Unemployed or student & 16 & 64 \\
\hline \multicolumn{3}{|l|}{ Household income per month } \\
\hline None & 12 & 48 \\
\hline$<\$ 60$ per month & 5 & 20 \\
\hline$\$ 60-300$ per month & 8 & 32 \\
\hline \multicolumn{3}{|l|}{ Vaginal sex during pregnancy } \\
\hline Yes & 24 & 96 \\
\hline \multicolumn{3}{|l|}{ Multiple partners in past year } \\
\hline $\operatorname{Yes}(>1)$ & 3 & 12 \\
\hline
\end{tabular}

High adherence defined as self-report PrEP use $\geq 25$ days in last 30 -days and never missed a PrEP prescription collection in pregnancy and postpartum period 
Another mother took PrEP because her child was still too young for her to get infected and she wanted to be there for the child. A few women also credited PrEP for their babies being born HIV-negative. "The baby was fine when I had her. She didn't have a problem. I trust that it was the pills I was taking." (PID152, 34 years).

Overall, women who adhered to PrEP expressed high satisfaction with PrEP feeling empowered to prevent HIV in their infants also contributing to their confidence in continuing use.

Partner HIV Risk and HIV Testing Women's fear of HIV acquisition among their child, and their own seroconversion, was frequently linked to women's perceived risk resulting from their partner's risky sexual behaviour.

Even if you sleep with your partner without protection [condoms] or if you don't know what he is up to, if you use PrEP, you know you are safe. That is the main reason that made me take PrEP ... He loves girls; He is not trustworthy. (PID156, 26 years)

Fear of partners' risky sexual behaviour was exacerbated by being pregnant and resulting limited willingness to have sex during this time.

When you are pregnant, you don't get to be with your partner a lot especially if you don't live together. So you don't know what he is doing and with whom. (PID 293, 20 years)

Known or perceived risky sexual behavior by their partners lead some women to believe their partner was likely HIV-infected. However, most women reported not knowing their partner's HIV status or their refusal to get tested. For example, "I took PrEP because of my partner's behavior. Based on how he behaves he can even bring disease to me." (PID114, 19 years).

Other women described forcing their partners to test, or not trusting them if they reported prior testing.

The people we live with nowadays are unfaithful. You would ask a person to go for HIV testing, they would lie and say they have but to find out that they didn't even go for testing. So, this (taking PrEP) is my way of protecting myself. (PID120, 32 years)

Other women stated they had tested with their partners in the past, but that their status may have changed due to their sexual behavior. Partner testing was infrequent and untrusted by women, especially those who knew their partners had other sex partners.

In addition, few women reported using condoms prior to PrEP start, or during PrEP use, in many cases because of their partner's choice.
He threw that condom away, because he was saying he will not use a condom. I told him this (condom use) was in the interest of our baby. Initially he thought I was cheating. (PID120, 32 years)

As a result, PrEP helped the women feel protected or safe when they had sex with their partners who they thought were risky partners, or when navigating condom use was difficult. Women's newfound sense of safety empowered them to continue to take PrEP. For example: "Now, I feel comfortable even when I don't use a condom. I tell myself that I am alright." (PID195, 25 years).

Community PrEP Encouragement Most women with longer-term, high PrEP adherence reported having disclosed their HIV status and their PrEP use to their family and/or sex partners. Encouragement from their primary partner, a friend or relative regarding their decision to start or continue taking PrEP was an important driver of consistent PrEP use.

She (mother) liked it and [said] that I should continue. Another person who knows is the father of my child, he knows that I am using PrEP. He didn't have a problem with it and said I should continue. I had made the decision, and no one was going to stop me. (PID067, 41 years)

Some were even interested in beginning PrEP themselves: "He asked how he could get it.....He even said that they should be available for everyone and not just pregnant people..." (PID195, 25 years).

A few women expressed concern about anticipated conflict or discouragement but were pleasantly surprised by their partners' responses.

I was concerned that my partner will tell me about the child. [Him questioning me about] taking a pill during pregnancy and how can I trust them [pills]. But I decided to be courageous and tell him because he will find out, he will see it on the [clinic] card. Luckily, it was received by someone who understood it. I told him about it the way I was told...He was not stubborn and said I should continue. (PID067, 41 years).

Others stated that their partner was concerned about the safety of taking PrEP during pregnancy and required additional information about PrEP to accept or support them:

...He didn't understand what PrEP was even when I explained it. He said I was taking pills that would endanger his baby, so we had a fight. He threw the pills in the bin. I spoke to them [PrEP counsellor] and they advised me on what I should do. 
They gave me a pamphlet and I sat him down and explained how it works. Only then did he believe that it won't endanger the child. (PID148, 35 years)

Several partners even helped remind women to take PrEP, this type of practical support may facilitate adherence: "sometimes he would remind me to take my tablets even before nine.... I won't lie he encourages me!" (PID147, 42 years).

Many participants received encouragement from relatives, particularly immediate family members like parents and siblings.

...My sister was happy for me. She said it was the first time she was hearing about it, but she said it sounded interesting when I explained it....Luckily, she had not heard about it and liked it, she said I should continue. (PID067, 41 years)

Others said they had personal agency, regardless of what their community said. Partner, family and friend encouragement following disclosure about taking PrEP was a significant driver in PrEP adherence.

\section{HIV-Related Factors}

Stigma Related to Taking PrEP Several women reported concerns about disclosing PrEP use because they feared, or had experienced, community stigmatization. One woman noted her fear of being assumed to be living with HIV: "I was concerned that they would say it is not PrEP....Saying that I am hiding, that [the pills] are something else [ART], that they are not for what I say they are for." (PID148, 35 years).

Other women supported this by reporting that on disclosing their PrEP use they were questioned about their HIV status. "They (friends) listened. They opened them.... They looked and asked why they look like ART. I said I was not told that they are ART." (PID178, 25 years).

Many women overcame that stigma by being open about their use of PrEP, and educating others in their lives about the benefits of taking PrEP. Despite some fear and reports of stigma, no participants reported that this affected their adherence. Most reported that in the end they were taking PrEP for themselves, not others.

\section{Concerns About PrEP Safety and Experiences of Side} Effects Pregnant women in this study who initiated PrEP at their first antenatal care visit early in pregnancy (median of 18 week's gestation; interquartile range [IQR] $=7$, 24 weeks). Several women reported experiencing side effects when they started PrEP, though it is difficult to distinguish this from normal pregnancy symptoms. Women expressed that the fear of future side effects motivated them to continue taking PrEP since they did not want to stop and restart PrEP and experience the same side effects.

It does take 2-3 days with any pill, having those side effects. Luckily, for me it only took one day. It (counselling) made me want to try it out myself and not hear about it from others. (PID067, 41 years)

When I started, I was vomiting so I thought I would stop taking it, but I thought, what will I do if I stop. I changed, I was taking it in the morning, I changed and took it at night, then I was fine. (PID043, 36 years)

Other participants were concerned about the safety of PrEP during pregnancy and breastfeeding, but were counselled on the risks and benefits. While women were concerned about, or experienced, transient side effects many continued taking PrEP because they did not want to go back to having side effects again. Women who reported high adherence were able to manage side effects and get over the transient side effects.

\section{Facility-Level Factors}

Participants learned about PrEP during the study and appreciated that education was integrated into ANC. In addition, participants appreciated the detailed counseling they received as part of the project.

They [project staff] also taught us about side effects that can manifest to some of us since our bodies are not the same. The explanation we get here is different from that we get in the clinics...they explained everything and that encouraged me to continue (on PrEP). (PID006, 16 years)

The counseling was also a key motivator for starting and staying on PrEP.

She (study counselor) said that when I use PrEP....

But what happened is, when I started using PrEP, I

had those things, feeling like I will vomit. But because

I was told about them, I knew I was not sick, it was just

the pills I was taking. (PID067, 41years)

All participants recommended continuing the offer of PrEP integrated into antenatal care. Some women made suggestions for other pregnant women: "I would tell her to take PrEP, because it has worked for me and that I have also started it while I was pregnant. Even my child was okay, I gave birth to a healthy child." (PID333, 23 years).

Participants liked that the offer of PrEP counseling and services were integrated into their antenatal and postnatal care services, and did not feel that the counseling or time spent was a burden on initiating or persisting on PrEP. 


\section{Discussion}

This study provides novel evidence on the facilitators of consistent PrEP use in pregnancy and postpartum. Individual level risk and risk perception encouraged women to start and stay on PrEP, especially perceived HIV risk related to partners' perceived sexual behaviors and limited knowledge about their serostatus, which increases the risk of vertical HIV transmission. Social support from families, partners and friends, were important contributors to daily adherence in pregnant and postpartum. Women in our study identified multiple barriers to PrEP adherence, including anticipated stigma and fear that people would think that PrEP was ART for HIV treatment. Women who were afraid of their partners' or friends' reactions managed these obstacles by disclosing their PrEP use upfront and providing information about PrEP benefits and safety during pregnancy. Women also reported concerns about safety and experiencing transient side effects. Despite these barriers, information, education from the PrEP study and empowerment of taking PrEP enabled women to overcome misgivings and continue taking and adhering PrEP. Finally, empathetic counsellors, and available PrEP services that were accessible and integrated into antenatal care, enabled women to start and continue taking PrEP, while ongoing adherence counselling improved women's ability to develop strategies to adhere to daily PrEP use.

Our findings have important implications for future maternal PrEP interventions. Participants highlighted women's fear of partners' risky behavior, and that partners were reluctant to test and share their serostatus. Secondary distribution of HIV self-tests, whereby female clients bring tests home to their partner(s), may be an effective way to promote HIV testing among male partners [24-27]. Further, prior research has demonstrated that partners are important barriers to women's participation in clinical trials, especially during pregnancy [28], and PMTCT activities more broadly [29]. Educating male partners about the safety and effectiveness of PrEP and providing HIV testing services for male partners may contribute to PrEP persistence and adherence for women during pregnancy and postpartum.

Most women in the study who reported high adherence had support from partners and/or family has they had disclosed their HIV status and PrEP use, and many benefitted from social support, encouragement and adherence reminders. Similar to ART programs, disclosure and family support appears to be key to longer-term PrEP adherence and persistence. A recent study from South Africa and Zimbabwe demonstrated that stigma and disclosure were significant concerns for young women initiating PrEP [30]. Our previous analysis demonstrated the role that anticipated stigma played as a barrier to PrEP initiation and persistence in our cohort [31]. Future interventions should address stigma, especially anticipated stigma, including mentor mother or peer based programs to share how women countered stigma and negativity in their community [32].

Pregnant women may experience more side effects than other PrEP users because of concomitant morning sickness and other hormonal changes, especially early on in pregnancy. Side effects were shown to affect PrEP continuation in pregnant women in Kenya [33] and should be addressed through education and counseling support. Women in our study were able to manage common side effects and persisted on PrEP. Some took PrEP at night when they were not prone to morning sickness. Others continued taking PrEP in postpartum period, despite abstinence during after childbirth, because they did not want to restart and experience side effects again.

Counseling about the risks, benefits, safety and adherence is essential to improving PrEP adherence, as others have demonstrated [34-36]. Women used the knowledge gained during counseling sessions to inform others about PrEP and were able to convince their partners and family to accept that PrEP was both safe and effective. Future programs should consider integrating HIV risk reduction counselling, including PrEP, by empathetic counsellors into existing antenatal and postnatal care.

Limitations include that the study was conducted in one urban facility in Cape Town, and may not represent rural perspectives or other regions of South Africa. Further, the study included postpartum women who returned for all of their PrEP dispensation visits and did not miss any PrEP visits, which may not be representative of other PrEP users. In addition, we did not use objective adherence measures of tenofovir to measure true PrEP adherence levels. Some women may misrepresent their adherence if they feel that the study staff would judge them (self-report bias). Other participants may return when they are postpartum to re-start PrEP for example when they resume sexual activity. Another important limitation is that this subset of women may differ from others in the study because of their ability to disclose their status and PrEP use to their partner and/or family, education, or relationship status, which may affect the generalizability of the results.

\section{Conclusions}

Our study identified important facilitators of optimal maternal PrEP use in pregnancy and postpartum. Individual risk, fear of HIV acquisition for self and infant, and partner sexual 
behaviors were important motivators to start PrEP and disclosure to family, partner and friends contributed to PrEP persistence. Maternal PrEP programs should consider how best to address barriers to PrEP use including stigma, fear around safety and side effects in the counseling around PrEP to ensure optimal PrEP use. Integration of PrEP, and adherence counseling, into existing antenatal and postnatal care services will help ensure access in pregnant and postpartum women at risk of HIV acquisition.

Acknowledgements We would like to thank our study participants, PrEP-PP study staff and the City of Cape Town Department of Health staff. We received the study drug $\left(\right.$ Truvada $\left.^{\circledR}\right)$ from Gilead (California, USA) and GeneXpert ${ }^{\circledR}$ STI test kits from Cepheid (California, USA). DJD received funding from Fogarty International Center and National Institute of Health (K01TW011187), TC and LM received funding from National Institute of Mental Health (R01MH116771).

Funding This study was supported through Grants from the National Institute of Mental Health (TC and LM; R01MH116771) and Fogarty International Center (DJD; K01TW011187).

\section{References}

1. Drake AL, Wagner A, Richardson B, John-Stewart G. Incident HIV during pregnancy and postpartum and risk of mother-to-child HIV transmission: a systematic review and meta-analysis. PLoS Med. 2014;11(2):e1001608.

2. Mofenson LM. Risk of HIV acquisition during pregnancy and postpartum: a call for action. J Infect Dis. 2018;218(1):1-4.

3. Mofenson LM. Tenofovir pre-exposure prophylaxis for pregnant and breastfeeding women at risk of hiv infection: the time is now. PLoS Med. 2016;13(9):e1002133.

4. Johnson LF, Stinson K, Newell ML, Bland RM, Moultrie H, Davies MA, et al. 2012 The contribution of maternal HIV seroconversion during late pregnancy and breastfeeding to motherto-child transmission of HIV. J Acquir Immune Defic Syndr. 1999;59(4):417-25.

5. Dinh TH, Delaney KP, Goga A, Jackson D, Lombard C, Woldesenbet $\mathrm{S}$, et al. Impact of maternal HIV seroconversion during pregnancy on early mother to child transmission of HIV (MTCT) measured at 4-8 weeks postpartum in South Africa 2011-2012: a national population-based evaluation. PLoS ONE. 2015;10(5):e0125525.

6. Wang L, Kourtis AP, Ellington S, Legardy-Williams J, Bulterys M. Safety of tenofovir during pregnancy for the mother and fetus: a systematic review. Clin Infect Dis. 2013;57(12):1773-81.

7. Ministry of Health NASCP. Guidelines on use of antiretroviral drugs for treating and preventing HIV infection in Kenya. Nairobi: Ministry of Health NASCP; 2016.

8. World Health Organization. WHO technical brief: preventing HIV during pregnancy and breastfeeding in the context of pre-exposure prophylaxis (PrEP). Geneva: World Health Organization; 2017.

9. Joseph Davey DL, Bekker LG, Gomba Y, Coates T, Myer L, Johnson LF. Modelling the potential impact of providing preexposure prophylaxis in pregnant and breastfeeding women in South Africa. AIDS. 2019;33(8):1391-5.

10. Joseph Davey DL, Pintye J, Baeten JM, Aldrovandi G, Baggaley R, Bekker LG, et al. Emerging evidence from a systematic review of safety of pre-exposure prophylaxis for pregnant and postpartum women: where are we now and where are we heading? J Int AIDS Soc. 2020;23(1):e25426.

11. Pyra M, Anderson PL, Hendrix CW, Heffron R, Mugwanya K, Haberer JE, et al. Tenofovir and tenofovir-diphosphate concentrations during pregnancy among HIV-uninfected women using oral preexposure prophylaxis. AIDS. 2018;32(13):1891-8.

12. Stranix-Chibanda L, Anderson PL, Kacanek D, Hosek S, Huang S, Nematadzira TG, Taulo F, Korutaro V, Nakabiito C, Masenya M, Lypen K, Brown E, Ibrahim ME, Yager J, Wiesner L, Johnston B, Amico KR, Rooney JF, Chakhtoura N, Spiegel HML, Chi BH; IMPAACT 2009 team. Tenofovir diphosphate concentrations in dried blood spots from pregnant and postpartum adolescent and young women receiving daily observed preexposure prophylaxis in sub-Saharan Africa. Clin Infect Dis. 2020:ciaa1872. https://doi.org/10.1093/cid/ciaa1872.

13. Joseph Davey DL, Daniels J, Beard C, Mashele N, Bekker LG, Dovel K, et al. Healthcare provider knowledge and attitudes about pre-exposure prophylaxis (PrEP) in pregnancy in Cape Town South Africa. AIDS Care. 2020;32(10):1290-4.

14. Joseph Davey D, Bekker LG, Coates TJ, Myer L. Contracting HIV or contracting SAR-CoV-2 (COVID- 19) in pregnancy? balancing the risks and benefits. AIDS Behav. 2020;24(8):2229-31.

15. Pintye J, Davey DLJ, Wagner AD, John-Stewart G, Baggaley $\mathrm{R}$, Bekker LG, et al. Defining gaps in pre-exposure prophylaxis delivery for pregnant and post-partum women in high-burden settings using an implementation science framework. Lancet HIV. 2020;7(8):e582-92.

16. Philbin MM, Parish C, Kinnard EN, Reed SE, Kerrigan D, Alcaide ML, et al. Interest in long-acting injectable pre-exposure prophylaxis (LAI PrEP) among women in the women's interagency HIV study (WIHS): a qualitative study across six cities in the United States. AIDS Behav. 2021;25(3):667-78.

17. Kuehn BM. Long-acting PrEP shows promise. JAMA. 2020;324(7):621.

18. Joseph Davey DL, Mvududu R, Mashele N, Lesosky M, Bekker LG, Khadka N, et al. Early pre-exposure prophylaxis (PrEP) discontinuation among pregnant and postpartum women: implications for maternal PrEP roll out in South Africa. medRxiv. 2021;7(8):e582

19. Braun V, Clarke V. Using thematic analysis in psychology. Qual Res Psychol. 2006;3(2):77-101.

20. Tong A, Sainsbury P, Craig J. Consolidated criteria for reporting qualitative research (COREQ): a 32-item checklist for interviews and focus groups. Int J Qual Health Care. 2007;19(6):349-57.

21. Ickovics JR, Meisler AW. Adherence in AIDS clinical trials: a framework for clinical research and clinical care. J Clin Epidemiol. 1997;50(4):385-91.

22. Corneli A, Perry B, Agot K, Ahmed K, Malamatsho F, Van Damme L. Facilitators of adherence to the study pill in the FEMPrEP clinical trial. PLoS ONE. 2015;10(4):e0125458.

23. Corneli A, Perry B, McKenna K, Agot K, Ahmed K, Taylor J, et al. 2016 participants' explanations for nonadherence in the FEM-PrEP clinical trial. J Acquir Immune Defic Syndr. 1999;71(4):452-61.

24. Thirumurthy H, Masters SH, Mavedzenge SN, Maman S, Omanga E, Agot K. Promoting male partner HIV testing and safer sexual decision making through secondary distribution of self-tests by HIV-negative female sex workers and women receiving antenatal and post-partum care in Kenya: a cohort study. Lancet HIV. 2016;3(6):e266-74.

25. Masters SH, Agot K, Obonyo B, Mavedzenge SN, Maman S, Thirumurthy H. Promoting partner testing and couples testing through secondary distribution of HIV self-tests: a randomized clinical trial. PLoS Med. 2016;13(11):e1002166. 
26. Choko AT, Corbett EL, Stallard N, Maheswaran H, Lepine A, Johnson CC, et al. HIV self-testing alone or with additional interventions, including financial incentives, and linkage to care or prevention among male partners of antenatal care clinic attendees in Malawi: an adaptive multi-arm, multi-stage cluster randomised trial. PLoS Med. 2019;16(1):e1002719.

27. Gichangi A, Wambua J, Mutwiwa S, Njogu R, Bazant E, Wamicwe J, et al. Impact of HIV self-test distribution to male partners of ANC clients: results of a randomized controlled trial in Kenya. J Acquir Immune Defic Syndr. 2018;79(4):467-73.

28. Ngure K, Trinidad SB, Beima-Sofie K, Baeten JM, Mugo NR, Bukusi EA, et al. The role of male partners in women's participation in research during pregnancy: a case study from the partners demonstration project. Reprod Health. 2017;14(Suppl 3):160.

29. McGrath CJ, Singa B, Langat A, Kinuthia J, Ronen K, Omolo $\mathrm{D}$, et al. Non-disclosure to male partners and incomplete PMTCT regimens associated with higher risk of mother-tochild HIV transmission: a national survey in Kenya. AIDS Care. 2018;30(6):765-73.

30. Velloza J, Khoza N, Scorgie F, Chitukuta M, Mutero P, Mutiti K, et al. The influence of HIV-related stigma on PrEP disclosure and adherence among adolescent girls and young women in HPTN 082: a qualitative study. J Int AIDS Soc. 2020;23(3):e25463.

31. Moran A, Mashele N, Mvududu R, Bekker LG, Gorbach P, Coates T, Myer L, Joseph Davey D. Maternal PrEP use in HIV-uninfected pregnant women in South Africa: Role of Stigma in PrEP initiation, retention and adherence. medRxiv 2020. https://doi.org/10. 1101/2020.11.24.20237867.
32. Akinde Y, Groves AK, Nkwihoreze H, Aaron E, Alleyne G, Wright $\mathrm{C}$, et al. Assessing the acceptability of a peer mentor mother intervention to improve retention in care of postpartum women living with HIV. Health Equity. 2019;3(1):336-42.

33. Kinuthia J, Pintye J, Abuna F, Mugwanya KK, Lagat H, Onyango $\mathrm{D}$, et al. Pre-exposure prophylaxis uptake and early continuation among pregnant and post-partum women within maternal and child health clinics in Kenya: results from an implementation programme. Lancet HIV. 2020;7(1):e38-48.

34. Pintye J, Beima-Sofie KM, Kimemia G, Ngure K, Trinidad SB, Heffron RA, et al. 2017 "I did not want to give birth to a child who has HIV": experiences using prep during pregnancy among HIV-uninfected Kenyan women in HIV-serodiscordant couples. J Acquir Immune Defic Syndr. 1999;76(3):259-65.

35. Pintye J, Drake AL, Kinuthia J, Unger JA, Matemo D, Heffron RA, et al. A risk assessment tool for identifying pregnant and postpartum women who may benefit from preexposure prophylaxis. Clin Infect Dis. 2017;64(6):751-8.

36. Pintye J, Kinuthia J, Roberts DA, Wagner AD, Mugwanya K, Abuna F, et al. 2018 brief report: integration of PrEP services into routine antenatal and postnatal care: experiences from an implementation program in Western Kenya. J Acquir Immune Defic Syndr. 1999;79(5):590-5.

Publisher's Note Springer Nature remains neutral with regard to jurisdictional claims in published maps and institutional affiliations. 